\title{
Recent aspects on outcomes in geriatric fracture patients
}

\author{
N. Suhm • D. Rikli • S. Schaeren • P. Studer • M. Jakob • \\ S. L. Kates
}

Received: 18 August 2010 / Accepted: 3 September 2010

(C) International Osteoporosis Foundation and National Osteoporosis Foundation 2010

\begin{abstract}
As the population ages, the number of fragility fractures is expected to increase dramatically. These injuries are frequently associated with less than satisfactory outcomes. Many of the patients experience adverse events or death, and few regain their pre-injury functional status. Many also lose their independence as a result of their fracture. This manuscript will explore problems and some potential solutions to evaluate the outcomes of geriatric fracture care. Specific, system-wide, and societal concerns will be discussed. Limited suggestions will be made for future steps to improve outcomes assessments.
\end{abstract}

Keywords Fractures - Geriatric fracture patients - HRQOL · Osteoporosis $\cdot$ Surgery

\section{Introduction}

Fractures of the hip, vertebrae, distal radius, and proximal humerus are the most frequent fractures in geriatric fracture patients [1]. Only few studies assess the overall impact on the patient's well-being following these injuries. This means that, at the beginning of the twenty-first century, we still lack very basic outcome data for the treatment in

N. Suhm $(\bowtie) \cdot$ D. Rikli $\cdot$ S. Schaeren $\cdot$ P. Studer $\cdot$ M. Jakob Basel University Hospital,

Basel, Switzerland

e-mail: nsuhm@web.de

\section{S. L. Kates}

University of Rochester School of Medicine and Dentistry, Rochester, NY, USA this group of patients. In our opinion, one major reason for this situation is related to the concept of "outcome" in geriatric fracture patients: an accepted definition or common understanding of this concept is still missing, and adequate instruments to quantify "outcome" in geriatric fracture patients are not routinely applied in the clinical setting.

The goal of this paper is to provide an in-depth view into three areas of concern regarding "Outcome in geriatric fracture patients". These concerns reflect the current situation mentioned above:

1. Morbidity and mortality are not adequate outcome parameters for assessment of operative therapy for disorders of the musculoskeletal system, especially in geriatric fracture patients. More comprehensive assessment and evaluation instruments are available to fulfill this task.

2. Many geriatric fracture patients are medically and surgically complex. It seems unlikely that improvements of a single element of fracture treatment- such as the implant - or a process - will result in significantly improved outcome of geriatric fracture patients. Instead, improvements in outcomes might be expected when the treatment process as a whole is looked upon as a subject of continuous reevaluation and adaptation.

3. Limitation of resources is a major problem of current healthcare systems [2]. Wise use of the existing resources is essential. Society will come to expect healthcare providers to seek ways to restore the geriatric fracture patients back to their pre-injury health and living status more frequently.

This manuscript will review and discuss these concerns. 
Table 1 Assessment tools

\begin{tabular}{ll}
\hline Individual assessment & Commonly used tests \\
\hline Cognitive assessment & Mini mental status exam \\
& Mini-cog \\
Functional assessment & Parker mobility score \\
& FIM, new mobility score \\
Nutritional assessment & Mini-nutritional score \\
Gait and balance & Timed up and go \\
& History \\
Level of illness & Charlson co-morbidity score \\
\hline
\end{tabular}

\section{Hypothesis 1: To measure "outcome" in geriatric fracture patients, additional and more appropriate assessment tools need to be applied}

Most clinical studies on geriatric fracture patients do not put the outcome into the broader context of health, function, and return to pre-injury living situation. Traditionally, physicians have measured the outcomes of health conditions by relying on mortality data. More recently, the international concern about health care outcomes has shifted toward the assessment of overall function. (Table 1)

The need here is for universal classification and assessment tools, both for function and health levels, in basic areas and roles of function in society. Some examples are listed below:

The International Classification of Functioning, Disability and Health, known as "ICF" was developed to provide a standard language and framework to describe and measure health and health-related states [3]. It is useful in organizing outcomes with respect to the various health effects experienced by patients and both the short- and long-term effects of the intervention. It can describe changes in body function and structure, what a person with a health condition can do in a standard environment (their level of capacity), as well as what they actually do in their usual environment (their level of performance).

Geriatric assessment refers to an overall evaluation of the health status of the elderly patient [4]. The well-being of any person relies upon many factors, only some of which are medical. Thus, an overall functional assessment is more holistic than a problem-based medical evaluation. The ultimate goal of such evaluations is to assess one's function.

Health-related quality of life (HRQOL) instruments attempt to measure the broad concept of health by assessment of difficulties with activities of daily living, including recreation, household management, difficulties in relationships with family, friends, and social groups. The HRQOL seeks to capture not only the ability to function within these roles but also the degree of satisfaction derived from doing them. The SF-36 and EQ-5D instruments are commonly used to assess HRQOL $[5,6]$.

\section{Assessment tools}

Another issue to discuss when looking at follow-up and outcome analysis is problems with self-administered evaluation instruments. The cognitively intact patients could participate in newly developed outcome measures such as the National Institutes of Health (NIH)-sponsored patientreported outcomes measurement information system (PROMIS) series of health outcomes instruments. "One main goal of the PROMIS initiative is to develop a set of publicly available computerized adaptive tests for the clinical research community" [7]. This instrument remains to be tested in the geriatric fracture population. Table 2 reviews commonly used in-hospital metrics for assessment of outcomes. Table 3 reviews societal assessment for the outcomes of geriatric fracture care.

It is well known that objective measures (i.e., radiographic appearance or healing of fracture sites) do not always correlate well to self-reported functional ability or health $[8,9]$. A similar pattern has been documented for the relationship between performance-based measures, such as the timed up and go [10] and self-reported measures of health status. Table 1 covers some commonly used individual assessment tools for geriatric fracture patients.

When designing future study protocols aiming towards geriatric fracture patients in general and towards hip fracture patients in particular, it is important to select appropriate evaluation instruments and good study design [11]. Future study planning should aim beyond the old, traditional mortality and morbidity measures or radiographic fracture union by including measures of function and assessment of overall health $[12,13]$.

When looking at the geriatric fracture patient itself, additional barriers toward improved assessment and outcome evaluation are identified. Most orthopedic clinical trials are not designed on the highest level of evidence [11], and more than $30 \%$ of the elderly patients have cognitive impairment. This means that a relevant subgroup among eligible patients cannot be included in high-quality studies when formal informed consent is required. Thus, we accept a selection bias by excluding such patients with cognitive impairment by means of the study protocol. There is a greater need for carefully designed, randomized, blinded controlled trials to

Table 2 Assessment of system in hospital

\begin{tabular}{ll}
\hline Hospital metric & Assessment technique \\
\hline Length of stay & Midnight census method \\
Mortality rate & All in-hospital deaths \\
Readmission rate & Readmissions with 30 days \\
Infection rate & Infections noted within 30 days \\
Financial results & Profit or loss; costs of care vs. national averages
\end{tabular}


Table 3 Outcomes evaluation at a societal level

\begin{tabular}{ll}
\hline Outcome question & Assessment method \\
\hline Incidence of fractures & Census and claims data \\
Mortality rate & Governmental death indices \\
Costs to system & Claims data analysis \\
Are services effective? & Large randomized controlled trials \\
Are services cost-effective? & Large RCT of services with costs of care \\
How can we improve quality? & RCT of different care models \\
How can we restore pre-injury function? & Unknown \\
\hline
\end{tabular}

answer many of the questions with the geriatric fracture population $[11,14]$. In addition, in order to make significant improvements with the treatment in geriatric fracture patients, more comprehensive assessment instruments must be applied to those patients.

\section{Hypothesis 2: Interprofessional and interdisciplinary care models will be implemented and must be evaluated for their impact on the geriatric fracture patient's outcome and on the socioeconomic costs}

Functional outcome in older fracture patients especially with hip fracture is disappointing $[15,16]$. Only $20 \%$ of hip fracture patients regain their pre-injury function. Fragility fractures in older adults describe two major problems: (1) osteoporotic fractures as a result of a low-energy trauma, and (2) age-related higher prevalence of co-morbidity, disability, and frailty, which puts patients at risk for postoperative complications and further fractures [16-21].

As many elderly patients are frail, clinical treatment is complicated by geriatric syndromes [20]. Thus, improved interdisciplinary care, with close cooperation between geriatricians and surgeons, was expected to result in better patient outcomes. This has been reported by some authors in the past several years [22-24]. The interdisciplinary program known as the "geriatric fracture center" approach starts interdisciplinary care preoperatively. Patient-centered protocol-driven patient care is used throughout the hospital stay, incorporating many team members (e.g., geriatrics, anesthesia, nursing, nutrition, social work, therapies, etc.) to improve overall patient care [25]. Further study of this type of collaborative effort is necessary to allow a better understanding of the elements of interdisciplinary care that are most important [26].

Interdisciplinary rehabilitative care has been recently evaluated with a meta-analysis by Handoll et al. in "The Cochrane Collaboration" [27]. They assessed multidisciplinary rehabilitation, which is defined as "services provided by a multidisciplinary team with the goal of reducing disability by improving task-oriented behaviors like walking and dressing." This study found "evidence that multidisciplinary rehabilitation is not harmful to the patients included into such programs" [27]. It is worthwhile to note that by the time that the rehabilitation process starts, many common problems, such as delirium, cardiac, and thrombotic complications, may have already occurred. Further study of the rehabilitative process with assessment of the patient's actual function seems necessary.

The critical components of this next generation of interdisciplinary care are system efficiency, the "culture" surrounding patient care, and scope of the interdisciplinary team. These new models of care will focus on the implementation of evidence-based treatment protocols along the clinical pathway which the geriatric fracture patient follows [28]. The standardized treatment protocols are patient-centered and the co-managed team individualizes the care. The process as a whole and treatment protocols are subject to a cycle of continuous quality improvement.

Despite early success with the GFC model, prospective multicenter studies with high patient volumes are needed - and are underway - to show translatability of this model into different healthcare systems and different settings [25, 26].

\section{Hypothesis 3: A comprehensive assessment of the geriatric fracture patient may influence decisions on his treatment and rehabilitation}

In clinical practice, there is limited time available to perform a comprehensive geriatric assessment on each fracture patient [4]. Reliability of the assessment may depend on the examiners. The assessment can be limited when being admitted to the emergency department as the geriatric fracture patients are often in a worse state of health than before injury. Many of the patients had fallen some hours prior and the assessment and the patients' responses to questions may not reflect their baseline status. A team approach to the geriatric patient is typically beneficial with the comprehensive geriatric assessment [4].

It appears that the quality of geriatric assessment is significantly improved when detailed information is gathered 
from a nursing home or assisted living facility [4]. In few cases do we talk with caregivers themselves or to relatives accompanying the patient through the emergency department.

The result from an individual geriatric assessment can be used to establish a baseline for future comparisons, establish diagnoses, monitor the course of treatment, provide prognostic information, implement treatment recommendations, and screen for occult conditions [4]. However, in order to become meaningful for treatment decisions other than emergency case and fracture stabilization, the assessment should be redone at least once during the hospitalization [4].

\section{Surgical decision making}

The decision process in geriatric fracture patients should include evidence-based classification and scoring systems that go beyond the well-known fracture classification system which are based on plain x-rays. Instead, the indication for the surgical treatment of fractures in an elderly patient must consider the individual's general health status, the specific pattern of injury, and the ability of the patient to actively participate in the rehabilitation process. Often, surgical treatment, discharge planning, and rehabilitation procedures are rarely based upon defined assessment procedures.

\section{Distal radius fractures}

The treatment paradigm for fractures of the distal radius has changed dramatically in the last 10 years. With few exceptions, these fractures were treated conservatively with closed reduction and immobilization with a plaster cast, regardless of fracture type, age, or functional demands. With better understanding of the biomechanics of wrist fractures including load transmission and pathomechanics of the different fracture types, surgery has become more desirable. Improved surgical approaches and the introduction of volar locking plates have shifted treatment of the distal radius fracture towards open reduction and internal fixation. These new treatment algorithms take more into account the specific fracture types, associated ligamentous lesions of the wrist, and the importance of restoration of the distal radioulnar joint. Enhanced preoperative imaging allows for better understanding of key fragments in articular fractures and for the development of individual preoperative planning. In most cases, after stable internal fixation, an early active mobilization program can be instituted. Fractures of the wrist are very common and are treated similar to other articular fractures. The restoration of articular congruency and alignment is achieved with stable internal fixation. The results published in the literature show more predictable functional restoration, less complications (e.g., chronic regional pain syndromes and loss of fixation), and better patient satisfaction [29, 30].

However, if surgery is feasible, does this suggest that it is good for the patient? Arora et al. recently report on a retrospective comparison of radiological and clinical results of patients over 70 years old with a fracture of the distal radius treated either by open reduction and internal fixation with a volar plate $(n=53)$ or conservatively by closed reduction and cast immobilization $(n=61)$ [31]. The radiographic results were significantly better in the operated group, but clinical outcome (range of motion, DASH, PRWE, and Green and O'Brien scores) did not differ, and pain level and number of complications were lower in the conservatively treated group. The authors concluded that an unsatisfactory radiographic outcome in elderly patients does not necessarily translate into an unsatisfactory functional outcome [31]. On the other hand, an elderly person with relevant functional impairment due to sequelae of a mal-united wrist fracture may lose his/her independence.

Treatment decisions for an elderly patient with a displaced distal radius fracture must not be based entirely on the radiological appearance of the fracture. A radiograph alone cannot predict which individual patient will take advantage of operative intervention [31]. Other criteria, e.g., activity level and mental state, have to be included in clinical decision making in order to determine the ideal treatment strategy for an individual patient.

\section{Proximal humerus fractures}

The proximal humerus fracture represents another area where decision making is difficult and is based on limited data. The elderly patient is often low demand, but may still rely upon the proximal humerus to maintain his/her independence. Use of the upper extremities when using assistive devices such as walkers may make the humerus a weight-bearing bone. Many of the proximal humerus fractures are minimally displaced and should be treated non-operatively. The displaced fractures are more difficult to treat. Percutaneous pins, nails, plates, and prosthetic replacements are all options for treatment [32, 33]. There is little evidence available to choose one therapy over another. It is often up to the surgeon to decide the appropriate therapy for the patient. The functional outcomes often will not match the radiographic appearance of the fracture at the conclusion of surgery [32-34]. Thus, the treatment that allows the patients to retain their independence should always be the preferred treatment.

\section{Vertebral fractures}

With vertebral fractures, the situation is more complex. Two-thirds of these fractures are morphologic and one-third are symptomatic $[35,36]$. Thus, fractures are frequently not 
diagnosed and not treated. This may create a significant amount of suffering for the patient. In those symptomatic cases, it remains uncertain whether operative or non-operative treatment is better. This situation was underscored by publication of randomized controlled trials that compared the outcomes after operative treatment of osteoporotic vertebral fractures by means of vertebroplasty with a sham procedure [37].

Hip fractures and controversies

Several current hip fracture controversies exist, and studies are being published regarding outcomes. It is widely accepted that fracture patterns which include reverse obliquity fractures, transtrochanteric fractures, fractures with a large posteromedial fragment implying loss of the calcar buttress, and fractures with subtrochanteric extension are to be looked upon as "unstable". These fractures, in general, should be treated with an intramedullary nail because of the more favorable biomechanical properties of a nail. However, in particular, it remains unclear which device to implant when fixing a pertrochanteric fracture $[38,39]$. Younger surgeons finishing training have been to shown to use the trochanteric entry nail as a primary device [40]. More senior surgeons particularly in the community setting often use the sliding hip screw as the preferred device for the same fracture [40]. Current studies find a little difference that exists between the devices for outcomes of radiographic union or complications [38, 40].

The sliding hip screw costs considerably less. None of the studies address the patient's functional outcomes however. This underscores the need for more function-based instruments to use when assessing the success of common treatments used in this patient population.

\section{Summary}

We present many questions but few answers to the initial concerns. To improve outcomes research, new geriatric patient outcome measures that quantify the return of functional status and pre-injury living situation need to be established. New instruments such as the NIH PROMIS tool may offer new opportunities for better outcomes research. We know that many geriatric patients do not regain their function or their health following injury. Comprehensive multidisciplinary programs offer some hope to improve this situation and high-quality clinical research will be needed to establish which models of care offer improvements for patients. Additionally, society expects physicians to improve the systems of care as care of the elderly fracture patients is proving to be a heavy burden on healthcare systems.
Conflicts of interest Norbert Suhm has received a research grant from Synthes and teaching fees from Synthes, AO, Lilly, MSD and Roche. Stephen Kates has received a research grant from Synthes and speaker's fees from Lilly. Daniel Rikli, Stephan Schaeren, Patrick Studer and Marcel Jakob declare that they have no conflicts of interest.

\section{References}

1. Court-Brown CM, Caesar B (2006) Epidemiology of adult fractures: a review. Injury 37(8):691-697

2. Kilgore ML et al (2009) Health care expenditures associated with skeletal fractures among Medicare beneficiaries, 1999-2005. J Bone Miner Res 24(12):2050-2055

3. WHO International Classification of Functioning, Disability and Health (ICF). International Classification of Functioning, Disability and Health (ICF) (2010) [cited 2010 6-5-2010]. http://www.who.int/ classifications/icf/en/.

4. Soriano RP (2007) The comprehensive geriatric assessment. In: Soriano RP (ed) Fundamentals of geriatric medicine. Springer, New York, pp 20-25

5. Hallberg I et al (2009) Health-related quality of life after vertebral or hip fracture: a seven-year follow-up study. BMC Musculoskelet Disord 10:135

6. Ekstrom W et al (2009) Quality of life after a subtrochanteric fracture: a prospective cohort study on 87 elderly patients. Injury 40(4):371-376

7. NIH. PROMIS.2010 [cited 2010 6-5-2010]. http://www.nihpromis. org/default.aspx.

8. Kooistra BW et al (2010) Outcomes assessment in fracture healing trials: a primer. J Orthop Trauma 24(Suppl 1):S71-S75

9. Dijkman BG et al (2010) When is a fracture healed? Radiographic and clinical criteria revisited. J Orthop Trauma 24(Suppl 1):S76-S80

10. Moore AA, Siu AL (1996) Screening for common problems in ambulatory elderly: clinical confirmation of a screening instrument. Am J Med 100(4):438-443

11. Bhandari M, Sprague S, Schemitsch EH (2009) Resolving controversies in hip fracture care: the need for large collaborative trials in hip fractures. J Orthop Trauma 23(6):479-484

12. Young Y, Fried LP, Kuo YH (2010) Hip fractures among elderly women: longitudinal comparison of physiological function changes and health care utilization. J Am Med Dir Assoc 11 (2):100-105

13. Hirose J et al (2010) Prediction of postoperative ambulatory status 1 year after hip fracture surgery. Arch Phys Med Rehabil 91 (1):67-72

14. Jeray KJ et al (2010) Are large fracture trials possible? J Orthop Trauma 24(Suppl 1):S87-S92

15. Kristensen MT et al (2010) Prefracture functional level evaluated by the New Mobility Score predicts in-hospital outcome after hip fracture surgery. Acta Orthop 81(3):296-302

16. Bentler SE et al (2009) The aftermath of hip fracture: discharge placement, functional status change, and mortality. Am J Epidemiol 170(10):1290-1299

17. Bundesamt für Gesundheit, Osteoporose und Stürze im Alter. Ein Public-Health-Ansatz, H.B.f.G. BAG, Editor. 2004: Bern.

18. Levers MJ, Estabrooks CA, Ross Kerr JC (2006) Factors contributing to frailty: literature review. J Adv Nurs 56(3):282-91

19. Rockwood K, Andrew M, Mitnitski A (2007) A comparison of two approaches to measuring frailty in elderly people. J Gerontol A Biol Sci Med Sci 62(7):738-743

20. Haentjens $P$ et al (2007) Survival and functional outcome according to hip fracture type: a one-year prospective cohort study in elderly women with an intertrochanteric or femoral neck fracture. Bone 41 (6):958-964 
21. Arinzon $Z$ et al (2005) Functional recovery after hip fracture in old-old elderly patients. Arch Gerontol Geriatr 40(3):327-336

22. Batsis JA et al (2007) Effects of a hospitalist care model on mortality of elderly patients with hip fractures. J Hosp Med 2 (4):219-225

23. Amatuzzi MM et al (2003) Interdisciplinary care in orthogeriatrics: a good cost-benefit model of care. J Am Geriatr Soc 51 (1):134-136

24. Khasraghi FA et al (2005) Effectiveness of a multidisciplinary team approach to hip fracture management. J Surg Orthop Adv 14 (1):27-31

25. Friedman SM et al (2008) Geriatric co-management of proximal femur fractures: total quality management and protocol-driven care result in better outcomes for a frail patient population. J Am Geriatr Soc 56(7):1349-1356

26. Friedman SM et al (2009) Impact of a comanaged Geriatric Fracture Center on short-term hip fracture outcomes. Arch Intern Med 169(18):1712-1717

27. Handoll $\mathrm{HH}$ et al (2009) Multidisciplinary rehabilitation for older people with hip fractures. Cochrane Database Syst Rev, (4): p. CD007125.

28. Beaupre LA et al (2006) Reduced morbidity for elderly patients with a hip fracture after implementation of a perioperative evidence-based clinical pathway. Qual Saf Health Care 15 (5):375-379

29. Gehrmann SV, Windolf J, Kaufmann RA (2008) Distal radius fracture management in elderly patients: a literature review. J Hand Surg Am 33(3):421-429

30. Osada D et al (2008) Prospective study of distal radius fractures treated with a volar locking plate system. J Hand Surg Am 33 (5):691-700
31. Arora R et al (2009) A comparative study of clinical and radiologic outcomes of unstable colles type distal radius fractures in patients older than 70 years: nonoperative treatment versus volar locking plating. J Orthop Trauma 23(4):237-242

32. Kontakis $G$ et al (2008) Early management of proximal humeral fractures with hemiarthroplasty: a systematic review. J Bone Joint Surg Br 90(11):1407-1413

33. Robinson CM, Christie J (1993) The two-part proximal humeral fracture: a review of operative treatment using two techniques. Injury 24(2):123-125

34. Owsley KC, Gorczyca JT (2008) Fracture displacement and screw cutout after open reduction and locked plate fixation of proximal humeral fractures [corrected]. J Bone Joint Surg Am 90(2):233240

35. Melton LJ 3rd et al (1989) Epidemiology of vertebral fractures in women. Am J Epidemiol 129(5):1000-1011

36. Cooper $C$ et al (1992) Incidence of clinically diagnosed vertebral fractures: a population-based study in Rochester, Minnesota, 1985-1989. J Bone Miner Res 7(2):221-227

37. Buchbinder R, Kallmes DF (2010) Vertebroplasty: when randomized placebo-controlled trial results clash with common belief. Spine J 10(3):241-243

38. Parker MJ and Handoll HH (2008) Gamma and other cephalocondylic intramedullary nails versus extramedullary implants for extracapsular hip fractures in adults. Cochrane Database Syst Rev, (3): p. CD000093.

39. Haidukewych GJ (2009) Intertrochanteric fractures: ten tips to improve results. J Bone Joint Surg Am 91(3):712-719

40. Forte ML et al (2010) Provider factors associated with intramedullary nail use for intertrochanteric hip fractures. J Bone Joint Surg Am 92 (5):1105-1114 